PoSTGRAD. MED. J., (1966), 42, 95

\title{
PROCEEDINGS OF THE CONFERENCE
}

\section{ON \\ “THE POSTGRADUATE MEDICAL CENTRE"}

ORGANIZED BY

\section{THE ASSOCIATION FOR THE STUDY OF MEDICAL EDUCATION}

Held at the University of Birmingham, on November 10th and 11th, 1965

\section{SUPPORTED BY SMITH KLINE AND FRENCH LABORATORIES, LTD.}

\author{
INTRODUCTION \\ J. R. ELLIS, M.B.E., M.D., F.R.C.P. \\ Secretary, Association for the Study of Medical Education.
}

The CONTExT of Postgraduate Medical Education in which this conference is set is one of active endeavour, rapid change and considerable excitement. At a time when there is much that is disturbing, uncomfortable and even depressing about medical affairs, postgraduate education stands out as an area in which there is rapid development, with new ideas being translated into action and in which hope is accompanied by achievement. The most obvious outward sign of all this is the Postgraduate Medical Centre. Over the past five years, and particularly in the last three, centre after centre has come into action in some part of England. It is difficult to put a precise figure to the number which now exist but in preparing for this conference we have found that there are well over 200 people in the country who hold positions as area organisers, clinical tutors, surgical tutors or psychiatric tutors.

A typical centre is situated in or near a regional board hospital and it has one of the consultants in charge. He has usually been appointed by the regional postgraduate medical committee but at the moment he is also the man who, with enthusiasm and enterprise, has taken the lead in this local area from the beginning. He is supported by some kind of local committee comprising many of his colleagues in his own hospital and usually some general practitioners and others from outside. The structure of the centre itself is either in temporary accommodation recently put up or in some converted part of an old hospital or occasionally in a new permanent structure. In all cases there is likely to be a large space for meetings or demonstrations with some kind of facilities for refreshment and perhaps a bar. There will be a library, some offices and, increasingly, a small amount of space for research and one or two rooms for seminars or smaller meetings.

Most centres provide a means whereby all the doctors of an area can come into contact with each other but the major function is an educational one. More often than not the first educational intent was to give better and more frequent refresher courses to general practitioners. This attempt to improve Continuing Education has been determined and maintained and has spread to include consultants, just as much as general practitioners. In addition there has been rapidly growing awareness of another, quite different, educational purpose. This is the provision of postgraduate training for pre-registration house officers and for registrars and senior registrars in training for the hospital specialties. In consequence each centre is now likely to be engaged upon quite a wide variety of educational activities, each aimed at a different group of doctors. Some are even concerned now in that most important of all activities, the provision of special vocational training for those who are going to enter general practice.

The finances of each centre come from three 
sources. Some money comes from the Regional Hospital Board, and since the publication by the Ministry of Health last year of the paper known as HM 64 (69) the amount of money coming from this source has considerably increased. In the long run it seems likely to be the major source. So far however many centres have profited more from funds provided by foundations, especially the Nuffield Provincial Hospitals Trust and King Edward's Fund. The third financial source has been a local appeal, made in the first instance to the doctors of the area, then to the local people and then to local industry. This third source is particularly important for several reasons. In some cases it has been the largest single source of finance and possibly over all the country it is still the most significant contribution. It shows that even after two decades of the welfare state it is possible, when the profession takes the lead, for local people to come enthusiastically to the support of the betterment of medicine. It also means that more often than not a medical centre is conscious of having succeeded by self help and of having something of its own which it has acquired by its own independent enterprise.

Within this outward appearance of similarity there is a wide variation between centres. Not even the title is the same everywhere and there are many different and at times confusing titles given to medical centres. The title of clinical tutor is not accepted everywhere and indeed it does not seem to be a very suitable one. Oddly enough the title "Director of Postgraduate Studies" is seldom used despite the fact that it is accurately descriptive. The local organisation for educational activity differs considerably from place to place. There are also differing degrees of interest in club activities. The constitution of centres varies greatly as does membership where this is laid down, and some centres require subscriptions whereas others do not. There are not only differing ideas on educational purposes but from place to place a different emphasis is placed on different parts of the total educational activity.

Many centres have special features. There is the Commonwealth school at Stoke. Regular residential courses are given to overseas and British graduates at Exeter. In some places successful courses for the primary fellowship have been given for some time, beginning with that in Southampton some years ago. The whole pattern of postgraduate preparation for the specialities is being changed by the courses for the Membership and Fellowship which are being successfully given for local registrars in places such as Dudley Road, Birmingham and South Mead, Bristol. Some centres are experimenting with television linkage. Plymouth is notable for its sound-broadcasting to local doctors. Canterbury and now Wessex are showing a most excellent example by carefully devised courses of preparation for general practice for those who have entered this field locally.

Most centres have established a successful symbiotic relationship with the B.M.A. Many have built up a close relationship with the local Faculty of the College of General Practice. Some have actually inaugurated a local medical society but the majority have absorbed a preexisting one. It is surprising how often a centre believes that it has absorbed the oldest medical society in the country. It is equally significant that not a few centres are convinced that they are the first in the country if not the only one still to exist. Despite this each centre is nevertheless part of a regional organisation. In this both the University and the Regional Hospital board play a part. There are some regions where the University is undoubtedly taking the lead, there are others where the regional board is the dominant partner. There are still other regions where neither is particularly active and a few where both work most energetically in close concert There are regions where for many years there has been an active postgraduate dean. There are others where this is a comparatively new post. There are areas where the influence of professorial units reach out far beyond the confines of the teaching hospital or of the special hospital. In some regions the rotation of registrars and senior registrars between teaching and regional board hospitals is well developed. There are other regions where this happens but little. Some regions have appointed psychiati ic tutors, others have appointed none. In most regions there is a tutor in surgery appointed by the Royal College of Surgeons of England and in some there are several of these.

While all this has been going on in what may be called "the periphery" there has been also a considerable degree of activity in relation to postgraduate medical education in what may be called "the centre". The Royal and other colleges have been taking a new look at their higher diplomas. Some have been beginning to define, others to redefine, the whole nature of postgraduate training for their branches of medicine. New consideration is being given to the duration, content, site and even methods of postgraduate training for the specialties. An attempt is beginning to analyse more clearly the purpose and methods of continuing education. 
Perhaps most important of all the Ministry of Health, accepting the importance of Postgraduate Training and Continuing Education to the standard of medical care, has begun to concern itself with the provision of facilities needed for these two parts of Postgraduate Medical Education.

Looking then at the national picture certain features emerge. There is growing awareness of the necessity for Continuing Education and of its dependence for success upon a sound undergraduate education. There is growing awareness also of the need for Postgraduate Training for everyone, including those who enter general practice. There is realisation therefore that the preparation for medicine now falls into three stages: A first undergraduate stage of a University education in medicine, a second stage of general vocational training represented now by a pre-registration year, and a third stage of special vocational training to fit a man for the practice of a particular branch of medicine.

It is apparent however that there has not been a central clarification of ideas leading to a firm national policy with clear cut objectives and definite plans for the provision of facilities, money, staff and organisation in the areas of actual activity. Instead there has been and still is almost wildfire peripheral activity. It is comparatively little related to any well defined central policy but it is undoubtedly healthy and exciting. The profession is leading, as indeed it should. One of the most powerful forces behind all this must surely be the degree to which contemporary medicine makes its practitioners desirous of being in contact with each other and of sharing critical discussion of all their work. Another important force must be the desire of hospital consultants, all of whom have had many years of experience of teaching, to give all they can to their juniors and a desire also to attract more and better junior staff by looking after them more carefully. A still further force must be the desire of general practitioners to come out of the isolation into which events have led them and to come back into the interdependent structure of medicine today. We must acknowledge also the tremendous stimulus which has come from the generous support of the Nuffield Provincial Hospitals Trust and the King Edward's Fund.

It is abundantly clear that we are in the early days of a transition period but already there is evidence of certain gaps in the total structure of postgraduate medical education, gaps which must soon be filled. The dual need for more money and more man-power is obvious but it is equally obvious that these are gaps that will not be quickly filled. Another very obvious need is for more cohesion both at the regional and at the national level. No centre will ever achieve and maintain full success in isolation. Each region must provide a good culture medium for its centres so that the necessary sustenance (of money, man-power and expertise, none of which are in unlimited supply) can find its way without waste to where it is most needed and can be most effective. Regionally and nationally we must achieve better co-ordination between all the bodies concerned: the medical school, the colleges and the departments of health. The dependence of postgraduate training upon an adequate structure of in-service training posts makes the fullest co-operation of the Ministry of Health vital to its success. The dependence of the standard of medical care upon postgraduate training and continuing education makes the provision of the necessary facilities for both as important a part of the health service as the provision of wards, out-patient clinics, operating theatres and health centres.

It is obvious that there are important contributions which only the University can make to postgraduate medical education. There are $\frac{0}{\varnothing}$ doubtless some people who feel that Universities should in fact take total responsibility for the $\frac{0}{7}$ whole of postgraduate medical education. However, very much of postgraduate training is vocational and it has not been the tradition of British Universities to concern themselves predominantly with vocational training. It would require a very remarkable change of attitude on the part of the British Universities for them to accept that it was part of their function to turn a medical graduate into an ear, nose and throat surgeon, or a cardiologist or a general practitioner. It would, on the other hand, be in keeping with the past tradition and present activity if we were to turn to the Royal colleges for a professional definition of the standards and nature of the special vocational training needed for the various branches of medicine. Irrespective however of which body, or bodies, defines the duration, content and site of postgraduate medical education it is obvious that there is a growing need for more knowledge and understanding of educational methods and far more effective exchange and economical use of educational techniques and facilities of all kinds. This is surely where the Association for the Study of Medical Education comes in.

ASME exists only for the betterment of medical education. It consists of corporate members and individual members. Corporate membership 
covers all the undergraduate medical schools, all the Royal and other colleges, nearly all the Regional Hospital Boards and Boards of Governors of Teaching Hospitals, and a wide number of other organisations concerned with medical education. Individual membership ranges through every branch of medical practice and medical science. ASME is therefore not only the Assocation of British and Commonwealth Medical Schools but it is also an Association of medical teachers and of those interested in and concerned with medical education. It is a professional body, linking together very many other professional bodies. It has no executive function. It can and does provide a service in educational expertise and facilities. It can and does conduct much needed research into medical education. It can and does provide facilities for free discussion on all matters relating to medical education. On the occasion of this conference the Association brings together people from many different parts of the country and from many different branches and organisations in medicine, so that they can discuss together under the auspices of an uncommitted professional body which has no axe to grind, the objectives, some of the methods and some of the problems of postgraduate medical education in the postgraduate medical centres.

\title{
First Session: Objectives and Methods
}

\section{Chairman: Professor D. V. Hubble}

\author{
INTERNS (Pre-Registration House Officers) \\ L. P. LE QUeSNE, D.M., M.Ch., F.R.C.S. \\ Professor of Surgery, The Middlesex Hospital Medical School
}

I MUST preface my remarks with two disclaimers. First, I wish to point out that I have no specialised knowledge of the subject on which I am to talk. My remarks are based on an interest in medical education, and my experience with house-surgeons in a general surgical unit in a teaching hospital. Secondly, I disclaim any responsibility for the use of the Transatlantic term "Interns" in the title of this communication. Aside from the fact that we have the long-established English term "houseman" (or housewoman) to describe the category of doctor under consideration, I am not certain that the duties and responsibilities of an intern are strictly comparable with those of a houseman, so that the term may be slightly misleading.

In considering the educational purpose and significance of the pre-registration appointment it is clear that this appointment must be related both to what comes before and that which follows afterwards. For purposes of convenience we are accustomed to talk of undergraduate training and postgraduate training, just as this afternoon we are discussing separately pre-registration house-jobs and the subsequent training to be either a consultant or a general practitioner. This division of the training of a doctor into apparently self-contained compartments is undoubtedly of convenience, but it is important that we do not allow our thinking on this subject to be shackled by what are, in fact, arbitrary divisions. For education is a continuous process. In some people this process continues, with everwidening horizons, up to the time they retire from active practice, whilst in others, perhaps because of the circumstances in which they practise medicine, it may terminate at a much earlier date. Similarly, there is great variation in the rate at which each individual's education progresses, so that different individuals collect different benefits at different times in the course. But these apparent discrepancies should in no way be allowed to obscure the essential fact that the education and training of a doctor is, or should be, a continuous process from the time he enters medical school, through the pre-registration year and on into postgraduate studies.

In relating the pre-registration posts to previous and subsequent training, two obvious points require emphasis. First, at the conclusion of the pre-registration year the careers of doctors immediately begin to show a marked divergence, some men going on to prepare themselves for careers as general practitioners, some as consultants, some for work overseas in various fields, and some for careers in many of the fields of so-called laboratory medicine. It is true that during their undergraduate and pre-registration days student-doctors show a variation in their 